\title{
Direct measurement of the singlet oxygen lifetime in zeolites by near-IR phosphorescence
}

\author{
Steffen Jockusch, ${ }^{a}$ J. Sivaguru, ${ }^{a}$ Nicholas J. Turro ${ }^{a}$ and V. Ramamurthy ${ }^{b}$ \\ a Department of Chemistry, Columbia University, 3000 Broadway, New York, NY, 10027, USA \\ ${ }^{b}$ Department of Chemistry, University of Miami, Coral Gables, FL, 33124-0431, USA
}

Received 2nd February 2005, Accepted 11th March 2005

First published as an Advance Article on the web 18th March 2005

\begin{abstract}
Time-resolved near-IR phosphorescence spectroscopy was employed to determine the lifetime of singlet oxygen in Y-zeolites and porous silica and it was found to depend strongly on the alumina content of the zeolite.
\end{abstract}

Zeolites are inorganic crystalline solids consisting of enclosed regular cavities or channels of well-defined size and shape, that are widely used in industry in separation processes, such as catalysts, and in nano-reactors. ${ }^{1-3}$ Molecular oxidations of hydrocarbons using zeolites as shape-selective catalysts have become popular. ${ }^{4-6}$ In the past several years, an increasing number of studies have been reported, showing that zeolite pores provide an environment for photo-induced oxidation of olefins with excellent selectivity. ${ }^{7-11}$ Many of these reported oxidations involve singlet oxygen $\left({ }^{1} \mathrm{O}_{2}\right)$, which was generated by photosensitization using sensitizer dyes. The sensitizer dye adsorbed on the zeolite surface (external or internal surface) after photoexcitation and intersystem crossing produces triplet states, which are quenched by ground state molecular oxygen (triplet oxygen, ${ }^{3} \mathrm{O}_{2}$ ) to generate singlet oxygen ${ }^{1} \mathrm{O}_{2}$. The singlet oxygen molecules diffuse through the zeolite pores to the substrate (e.g. olefin) and oxidation can occur. The lifetime of singlet oxygen varies many orders of magnitude depending on its environment. ${ }^{12}$ If singlet oxygen is quenched by the zeolite surface during the diffusion process, oxidation of the substrate may be inefficient. Therefore, the knowledge of the lifetime of singlet oxygen in zeolites is essential for an understanding of the mechanism of the oxidation process. To the best of our knowledge, except for one product-based estimate, ${ }^{13}$ the lifetime of singlet oxygen in zeolites has not been reported. Pace and Clennan estimated the upper limit of the lifetime of singlet oxygen from product yields of the oxidation of 2-methyl-2-heptene on methylene blue doped $\mathrm{NaY}$ zeolite as $7.5 \mu \mathrm{s} .{ }^{13}$ Because the product-based studies can give only an estimate of the upper limit based on several assumptions, a direct spectroscopic-based lifetime of ${ }^{1} \mathrm{O}_{2}$ in zeolites is desirable. In this report we employed time-resolved phosphorescence spectroscopy to determine the singlet oxygen lifetime in different zeolites, and compare the results to the lifetime of ${ }^{1} \mathrm{O}_{2}$ in porous silica.

For our studies we selected the ketones benzophenone, xanthone, and thioxanthone (Table 1) as sensitizers of singlet oxygen, because of their high triplet quantum yield and their photostability. ${ }^{14}$ Furthermore, these ketones possess good absorption properties at $355 \mathrm{~nm}$, the emission wavelength of a frequency tripled Nd:YAG laser, which we used to excite the sensitizer. In addition, we selected the sensitizer dye thionine, which is similar in structure to methylene blue, a popular singlet oxygen sensitizer, but has a strong absorption at $532 \mathrm{~nm}$, the wavelength of a frequency doubled Nd:YAG laser. Popular zeolites for oxidations are Y-zeolites $\left[\mathrm{Na}_{x}\left(\mathrm{AlO}_{2}\right)_{y}\left(\mathrm{SiO}_{2}\right)_{z}\right]$, consisting of accessible supercages (diameter $c a .13 \AA$ ) and entry windows of $8 \AA$. The zeolites were activated and the sensitizers were loaded (one sensitizer molecule per five supercages) using standard methods published previously. ${ }^{7,11,15,16}$ Particular care was taken to remove water traces from the zeolite samples by pumping to a vacuum of $1 \times 10^{-5}$ Torr for at least two hours prior to the measurements. Water is known to be an efficient singlet oxygen quencher. $^{12}$

Singlet oxygen possess a phosphorescence centered at $1270 \mathrm{~nm},{ }^{17}$ which we employed as an analytical tool to measure singlet oxygen lifetimes in zeolites directly. The singlet oxygen phosphorescence decay traces were measured as follows: zeolite samples in a $0.2 \times 1 \times 4 \mathrm{~cm}$ quartz cell were irradiated with pulses $\left(355 \mathrm{~nm}\right.$ or $532 \mathrm{~nm}, 10 \mathrm{~ns}$, ca. $5 \mathrm{~mJ}$ pulse $^{-1}$ ) from a Spectra Physics Nd:YAG laser (GCR-150-30). The singlet oxygen phosphorescence at $1270 \mathrm{~nm}$ was collected and isolated using a lens and filter system (interference filter, NB-170-010-B, Spectrogon) and focused into a NIR sensitive PMT (H9170-4, Hamamatsu). The photocurrent from the PMT was amplified (SR 560 or SR 445, Stanford Research Systems) and stored on a digital oscilloscope (TDS 360, Tektronix). Signal rise times of $\approx 50$ and $\approx 500$ ns were achieved using the amplifier SR 445 or SR 560, respectively.

Fig. 1 shows representative luminescence decay traces at $1270 \mathrm{~nm}$ obtained after laser excitation of benzophenone loaded $\mathrm{NaY}(\mathrm{Si} / \mathrm{Al}=2.4)$. At an oxygen pressure of $1 \mathrm{~atm}$, a strong luminescence signal was observed (Fig. 1, left). Conversely, the luminescence signal at $1270 \mathrm{~nm}$ was negligible after removal of oxygen from the sample (vacuum of $1 \times 10^{-5}$ Torr). A short component luminescence remained, which is probably caused by scattered light from the excitation laser pulse. The strong, long-lived luminescence was recovered after the sample cell was refilled with oxygen $(1 \mathrm{~atm})$. Subtraction of both decay traces from each other, the trace at $1 \mathrm{~atm}$ oxygen and the trace under vacuum (Fig. 1, left), yielded the phosphorescence decay trace of singlet oxygen (Fig. 1, right). The trace fitted to first-order kinetics and showed a lifetime of $7.9 \mu$ s. Pace and Clennan estimated a lifetime of singlet oxygen in $\mathrm{NaY}(\mathrm{Si} / \mathrm{Al}=2.4)$ of $7.5 \mu \mathrm{s}$ from product studies (indirect method) ${ }^{13}$ which is in good agreement with our singlet oxygen phosphorescence lifetime $(7.9 \mu \mathrm{s})$ (direct method).
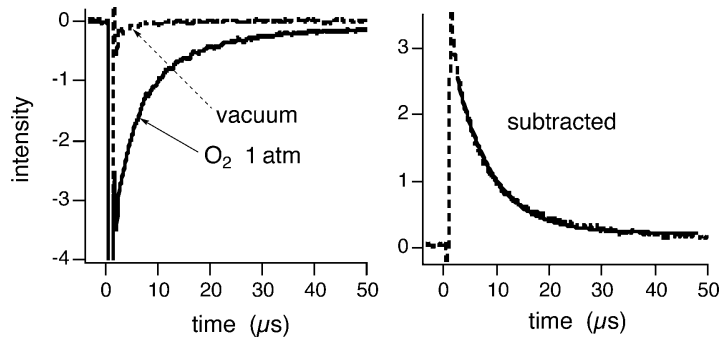

Fig. 1 Left: luminescence decay traces observed at $1270 \mathrm{~nm}$ after excitation of benzophenone adsorbed on $\mathrm{NaY}(\mathrm{Si} / \mathrm{Al}=2.4)$ under vacuum and 1 atm $\mathrm{O}_{2}$. Right: the subtracted signal corresponds to the phosphorescence decay trace of singlet oxygen. Dashed line: experimental data; solid line: fit to a first-order decay kinetic.

In order to investigate the influence of alumina (or associated cations) in the zeolite framework on the singlet oxygen lifetime, $\mathrm{NaY}$ with a lower alumina content was used $(\mathrm{Si} / \mathrm{Al}=80)$. 
Table 1 Lifetime of singlet oxygen $(\tau)$ generated by sensitization with different sensitizers loaded into Y-zeolites of different ratio of Si and $\mathrm{Al}$ or a porous silica

$\tau^{1} \mathrm{O}_{2} / \mu \mathrm{s}^{c}$
$7.9 \pm 0.1$

${ }^{a}$ Excitation at $355 \mathrm{~nm}$; loading: 1 sensitizer molecule per 5 supercages. ${ }^{b}$ Excitation at $532 \mathrm{~nm}$; loading: 1 sensitizer molecule per 150 supercages. ${ }^{c}$ Statistical error limits from single exponential fit of decay curves.

A significantly longer singlet oxygen lifetime was observed (35 $\mu$ s; Table 1). Porous silica adsorbs sensitizers in a manner similar to zeolites, but contains no alumina or cations. An even longer singlet oxygen lifetime was observed in porous silica (64 $\mu \mathrm{s}$; Table 1), which is consistent with a quenching role of the alumina anions (or associated cations). Iu and Thomas reported lifetimes of singlet oxygen in silica slurries in organic solvents (benzene and cyclohexane) from 13 to $18 \mu$ s using pyrene and 2-acetonaphthone as sensitizers. ${ }^{18,19}$ The authors attributed the short singlet oxygen lifetime to quenching by adsorbed water and silanol groups on the silica surface. In our experiments we used dry silica powder, which was activated at $500{ }^{\circ} \mathrm{C}$ prior to sensitizer loading and pumped to a vacuum of $1 \times 10^{-5}$ Torr for at least two hours prior to the measurements. The absence of solvent and the careful water removal probably caused our longer singlet oxygen lifetime $(64 \mu \mathrm{s})$. The quenching role of water was verified by addition of $\approx 20 \%$ water $(\mathrm{w} / \mathrm{w})$ to a zeolite sample $(\mathrm{NaY} ; \mathrm{Si} / \mathrm{Al}=80)$ loaded with benzophenone. The singlet oxygen phosphorescence was quenched in the presence of water compared to the same sample before addition of water.

To investigate the influence of the sensitizer, zeolite samples were loaded with different sensitizers: xanthone, thioxanthone, and thionine. The observed singlet oxygen lifetimes are shown in Table 1. The lifetimes are slightly shorter than those for the zeolites containing benzophenone as sensitizers. The differences in lifetime are assigned to singlet oxygen quenching by the sensitizers. In addition, trace amounts of water, which are difficult to remove, will reduce the singlet oxygen lifetime.

In summary, to the best of our knowledge, the lifetime of singlet oxygen in zeolites was determined for the first time by time resolved phosphorescence (direct method). The observed lifetime of singlet oxygen depends strongly on the alumina content of the zeolite framework; e.g. in $\mathrm{NaY}(\mathrm{Si} / \mathrm{Al}=2.4)$ a lifetime of $7.9 \mu \mathrm{s}$, in $\mathrm{NaY}(\mathrm{Si} / \mathrm{Al}=80)$ a lifetime of $35 \mu \mathrm{s}$, and in porous silica (no alumina and cations present) a lifetime of $64 \mu \mathrm{s}$ was observed. In addition to the alumina anions, the cations associated with the alumina probably play an important role in the quenching of singlet oxygen. A detailed study of the dependence of the lifetime of singlet oxygen on factors such as the type of exchangeable cations, sensitizers, water content, and oxygen concentration is currently in progress and subject of an upcoming publication.

\section{Acknowledgements}

This work was supported by the National Science Foundation (Grant CHE 04-15516).

\section{References}

1 D. W. Breck, Zeolite Molecular Sieves, R. E. Krieger, Malabar, FL, 1984.

2 A. Dyer, An Introduction to Zeolite Molecular Sieves, Wiley, New York, 1988.

3 H. Van Bekkum, E. M. Flaningen, and J. C. Jansen, Introduction to Zeolite Science and Practice, Elsevier, Amsterdam, 1991.

4 E. L. Clennan, Molecular Oxygenation in Zeolites, Mol. Supramol. Photochem., 2003, 9, 275-308.

5 S. Vasenkov and H. Frei, Photooxidation in Zeolites, Mol. Supramol. Photochem., 2000, 5, 295-323.

6 C.-H. Tung, L.-Z. Wu, L.-P. Zhang, H.-R. Li, X.-Y. Yi, K. Song, M. Xu, Z.-Y. Yuan, J. Q. Guan, H.-W. Wang, Y.-M. Ying and X.-H. $\mathrm{Xu}$, Microreactor-controlled Selectivity in Organic Photochemical Reactions, Pure Appl. Chem., 2000, 72, 2289-2298.

7 J. Shailaja, J. Sivaguru, R. J. Robbins, V. Ramamurthy, R. B. Sunoj and J. Chandrasekhar, Singlet Oxygen Mediated Oxidation of Olefins within Zeolites: Selectivity and Complexities, Tetrahedron, 2000, 56, 6927-6943.

8 M. Stratakis, D. Kalaitzakis, D. Stavroulakis, G. Kosmas and C. Tsangarakis, Remarkable Change of the Diastereoselection in the Dye-Sensitized Ene Hydroperoxidation of Chiral Alkenes by Zeolite Confinement, Org. Lett., 2003, 5, 3471-3474.

9 E. L. Clennan, J. P. Sram, A. Pace, K. Vincer and S. White, Intrazeolite Photooxidations of Electron-Poor Alkenes, J. Org. Chem., 2002, 67, 3975-3978.

10 C.-H. Tung, H. Wang and Y.-M. Ying, Photosensitized Oxidation of Alkenes Adsorbed on Pentasil Zeolites, J. Am. Chem. Soc., 1998, 120, 5179-5186. 
$11 \mathrm{X} . \mathrm{Li}$ and V. Ramamurthy, Selective Oxidation of Olefins within Organic Dye Cation-exchanged Zeolites, J. Am. Chem. Soc., 1996, 118, $10666-10667$.

12 R. Schmidt and E. Afshari, Collisional Deactivation of $\mathrm{O}_{2}$ by Solvent Molecules. Comparative Experiments with ${ }^{16} \mathrm{O}_{2}{ }^{18} \mathrm{O}_{2}$, Ber. BunsenGes. Phys. Chem., 1992, 96, 788-794.

13 A. Pace and E. L. Clennan, A New Experimental Protocol for Intrazeolite Photooxidations. The First Product-based Estimate of an Upper Limit for the Intrazeolite Singlet Oxygen Lifetime, J. Am. Chem. Soc., 2002, 124, 11236-11237.

14 N. J. Turro, Modern Molecular Photochemistry, University Science Books, Sausalito, CA, 1991

15 J. Shailaja, P. H. Lakshminarasimhan, A. R. Pradhan, R. B. Sunoj, S. Jockusch, S. Karthikeyan, S. Uppili, J. Chandrasekhar and N. J. Turro, Alkali Ion-controlled Excited-state Ordering of
Acetones Included in Zeolites: Emission, Solid-State NMR, and Computational Studies, J. Phys. Chem. A, 2003, 107, 3187-3198.

16 V. Ramamurthy, D. R. Sanderson and D. F. Eaton, Control of Dye Assembly within Zeolites: Role of Water, J. Am. Chem. Soc., 1993, 115, 10438-10439.

17 A. U. Khan and M. Kasha, Direct Spectroscopic Observation of Singlet Oxygen Emission at $1268 \mathrm{~nm}$ Excited by Sensitizing Dyes of Biological Interest in Liquid Solution, Proc. Natl. Acad. Sci. USA, 1979, 76, 6047-6049.

18 K. K. Iu and J. K. Thomas, Quenching of Singlet Molecular Oxygen $\left({ }^{1} \Delta_{g} \mathrm{O}_{2}\right)$ in Silica Gel/Cyclohexane Heterogeneous Systems. A Direct Time-resolved Study, J. Am. Chem. Soc., 1990, 112, 3319-3325.

19 K. K. Iu and J. K. Thomas, Quenching of Singlet Molecular Oxygen $\left({ }^{1} \Delta_{\mathrm{g}} \mathrm{O}_{2}\right)$ in Silica Gel-solvent Heterogeneous Systems II. A Direct Time-resolved Study, J. Photochem. Photobiol., A, 1993, 71, 55-60. 